\title{
Imaging of the knee
}

\section{Common acute presentations to general practice}

Ursula Ridley, Lloyd Ridley

\section{Background}

Knee pain is a common symptom in the community. There is a wide range of conditions that can cause pain. Identifying the type and severity of the condition is important for effective management. There are several guidelines for the appropriate imaging of patients presenting with knee pain. Presentation is generally divided into a post-traumatic group and those without known trauma (including arthritis).

\section{Objective}

The aim of this article is to discuss the approach to imaging of the knee with reference to Diagnostic Imaging Pathways and illustrate some of the conditions that may be encountered.

\section{Discussion}

The initial workup is typically done in general practice to determine which patients require referral for specialist management and which patients can be cared for in the community. Most cases presented in this article are patients referred from primary care to a metropolitan radiology practice during a six-month period, and they represent examples of some of the more common pathologies.
KNEE PAIN affects $25 \%$ of adults, and the prevalence has increased by $65 \%$ in the past 20 years. ${ }^{1}$ Many patients with pain have a history of trauma. In older patients, acute pathology is usually superimposed on chronic degeneration/osteoarthritis.

This article focuses on post-traumatic pain and monoarticular arthritis, describing some of the common findings and disease patterns shown on imaging.

While there are several guidelines for the appropriate use of imaging, the Australian website Diagnostic Imaging Pathways will be used as the standard for this narrative. ${ }^{2}$

\section{Post-traumatic knee pain}

Of all joints, the knee is most commonly affected by sports injuries, reducing the quality of life in young and physically active patients. Injuries typically occur either because of a direct force to the lower limb or as a result of a sudden change of direction. In the latter scenario, the foot is planted while the weight of the body and muscle action results in a twisting and valgus or varus force. By convention, one describes what is happening to the distal portion (ie tibia with respect to the femur). Valgus describes lateral movement; varus describes medial movement. The valgus/ rotation mechanism is often called the 'pivot shift' injury.

In a large Swedish study of major knee injuries, anterior cruciate ligament (ACL) rupture was the most common structural injury (52\%), followed by meniscal tear (41\%) and lateral patella dislocation (LPD, 17\%). However, individuals aged $\leq 17$ years were more likely to have LPD, followed by meniscal tear in boys and ACL tear in girls. ${ }^{3}$

\section{The role of $\mathrm{X}$-rays in knee trauma}

Clinical assessment, aided by tools such as the Ottawa knee rules (Box 1), considers the likelihood of fracture. ${ }^{4}$ When a fracture is suspected, an X-ray is recommended as the initial investigation (Figure 1).

The fractures likely to be seen in general practice are often relatively subtle on $\mathrm{X}$-rays. One example is depressed fractures of the tibial plateau (Figures 2A and 2B). There are several 'avulsion' fractures that indicate significant joint derangement. Important examples are the Segond fracture (avulsion from the lateral aspect of the tibial plateau) and the 'arcuate sign' (avulsion of the tip of the fibula). Providing the radiologist with an accurate history of the mechanism of injury and

\section{Box 1. The Ottawa knee rules 4}

Knee X-ray indications after acute knee injury include:

- age $\geq 55$ years

- tenderness at the head of the fibula

- isolated tenderness of the patella

- inability to flex knee to 90 degrees

- inability to bear weight (defined as an inability to take four steps, ie two steps on each leg, regardless of limping) immediately and at presentation.

Reproduced with permission of The Royal Australian College of General Practitioners from Yao K, Haque T, The Ottawa knee rules: A useful clinical decision tool, Aust Fam Physician 2012;41(4):223-24. 


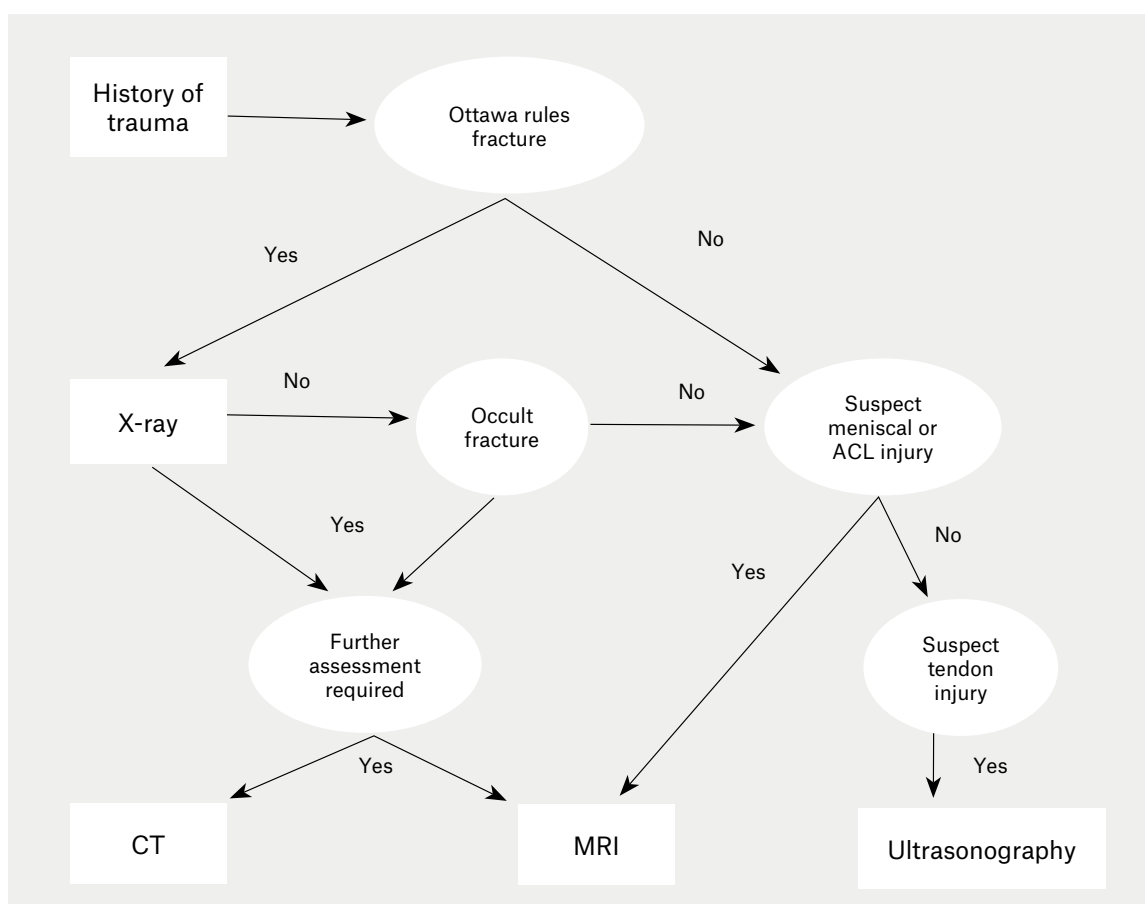

Figure 1. A simplified diagnostic algorithm for imaging of the knee after an acute injury Note: Non-radiological options are not included.

$A C L$, anterior cruciate ligament; $C T$, computed tomography; MRI, magnetic resonance imaging
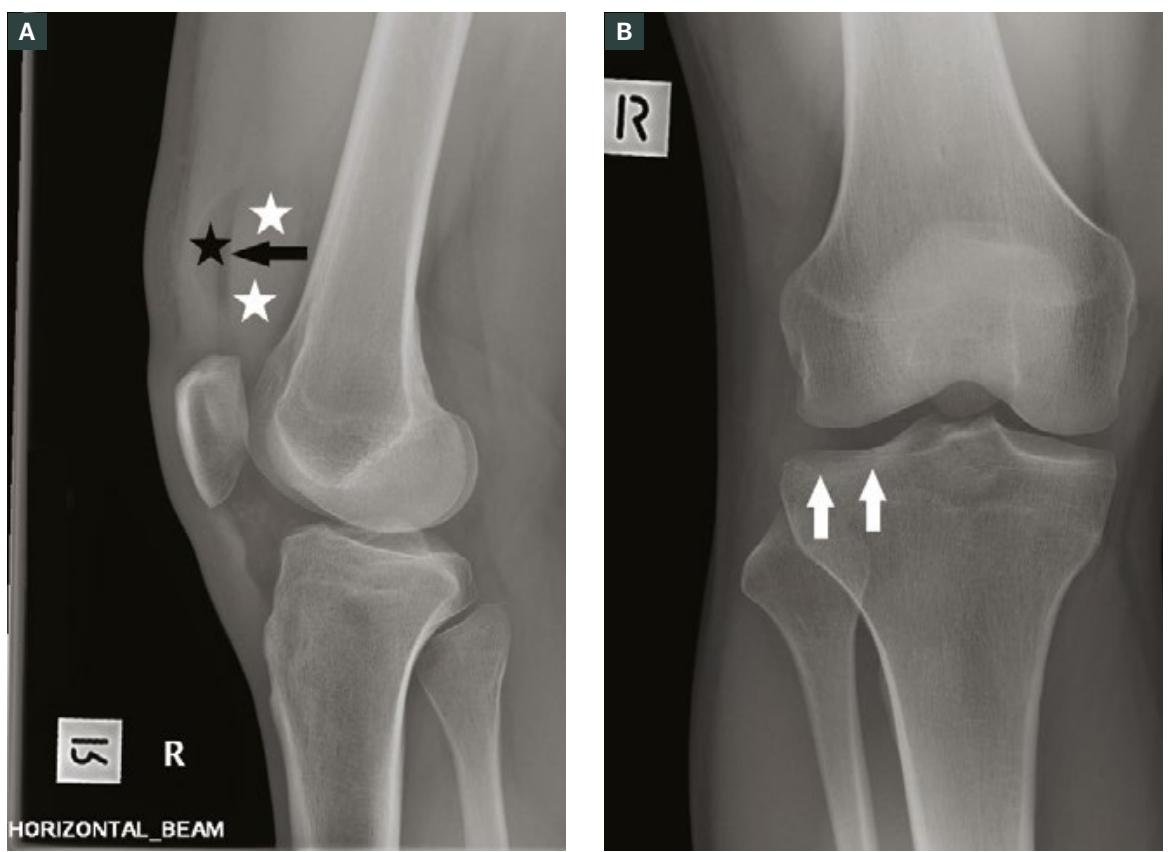

Figure 2. A. Lipohaemarthrosis in the suprapatellar recess with fat (black star) and fluid (white stars) producing a fluid level (black arrow). Note that this image is taken with a horizontal beam; B. Frontal $\mathrm{X}$-ray shows a relatively subtle lateral tibial plateau fracture (white arrows) that might be overlooked if the lipohaemarthrosis was not recognised.

Note: These images are both of the same patient. site of maximum tenderness can help to direct the radiologist's search for these findings and determine which findings are clinically relevant.

A direct blow to the patella typically causes a comminuted fracture. Indirect forces - typically forceful quadriceps contractions when falling feet first - are associated with transverse fractures through the body of the patella or patella tendon. Patella dislocation results in shear fractures of the articular surface. ${ }^{5}$

A knee effusion is an indirect sign of injury. On X-ray it is best seen in the suprapatellar recess. Of importance is the recognition of a fat-fluid level in the effusion, known as a lipohaemarthrosis (Figure 2A). This represents fat that has escaped from the bone marrow into the joint, which is a specific sign indicating an intra-articular fracture.

\section{The role of ultrasonography in acute trauma}

Where clinical examination indicates that a fracture is unlikely but signs point to a superficial structure, particularly the extensor mechanism (patella tendon or quadriceps tendon), the Australian guidelines recommend ultrasonography as the primary investigation. ${ }^{2}$

Ultrasonography is an inexpensive, time-efficient and safe method for assessing superficial structures. It will also detect a joint effusion. One limitation of ultrasonography is the limited view, which makes the examination difficult in obese or muscular patients, reducing its accuracy. Tendon ruptures are also relatively rare and not always clinically considered. ${ }^{6}$

\section{The role of computed tomography}

Computed tomography (CT) is useful for characterising the type of fracture. It is primarily indicated either when an $\mathrm{X}$-ray is normal but the clinical features remain suggestive of fracture, or to further characterise a fracture that has been visualised on an X-ray. In the general practice setting, the search for an occult fracture with CT will generally occur when magnetic resonance imaging (MRI) is not available - typically for patients aged $\geq 50$ years who are suspected of having 
an insufficiency fracture and who are ineligible for a rebatable scan covered by the Medicare Benefits Schedule (MBS).

\section{The role of magnetic resonance imaging}

MRI provides excellent detail of the soft tissues of the knee (including ligaments, menisci and articular cartilage). Bone marrow oedema is a key indicator of subtle bone injuries such as microtrabecular fractures. As a result, MRI is the examination of choice for the evaluation of post-traumatic bone pain or instability. ${ }^{2} \mathrm{~A}$ high diagnostic yield can be expected in young patients (aged $<50$ years). A negative MRI can reassure patients. When positive, the scan enables earlier targeted management.

In Australia, the indications for studies that are covered by the MBS are limited (Box 2).

\section{Cruciate ligament injuries}

ACL injuries are well studied in sports medicine, with women generally at higher risk than men. Sports with frequent contact (such as football and rugby) and those with high-impact rotation on landing (such as gymnastics) have the highest rates at approximately 2-5 injuries per 10,000 playing hours. ${ }^{7}$ ACL tears may be partial or complete. Only $12 \%$ of ACL tears are isolated, with meniscal tears being the most common associated injury. ${ }^{3}$

MRI is the best imaging modality to assess the ACL and identify associated injuries (Figures 3A and 3B). The types of co-existent injuries are dependent on the mechanism of injury. In a 'pivot shift' injury, the ACL injury is characteristically associated with bone marrow changes ('bone bruise') in the lateral femoral condyle and posterolateral tibial plateau as a result of the associated impaction injury of these bones. Patients with both an ACL injury and disruption of the 'posterolateral corner' (which includes the posterolateral capsule and the popliteus muscle) or 'posteromedial corner' (which includes the posterior horn of the medial meniscus and the posterior oblique ligament) are more likely to have knee instability. As a result, the radiological report will often mention these structures.
The posterior cruciate can also be injured, usually in a hyperextension injury of the knee (Figure 4).

\section{Meniscal tears}

In younger patients (aged $<50$ years), meniscal tears typically occur as a result of twisting movements of the knee when under load (weight bearing). In older patients (aged $\geq 50$ years), tears are more commonly a result of degeneration. MRI is useful for detecting and characterising meniscal tears (Figure 5). Tears near the attachments ('root') of the meniscus, and those which are displaced (such as buckethandle tears and free fragments), are more liable to cause locking and therefore should be referred to a surgeon.

\section{Patellofemoral joint abnormalities}

Patellofemoral joint abnormalities are a common cause of pain. The pain is typically described as being worse when bending the knee, such as during stair climbing or driving. A feeling of knee instability or history of patellar dislocations may also be described. Abnormal tracking and/or overuse can increase cartilage damage (chondromalacia patella). Acute dislocations result in shear injuries of the articular surfaces of either the patella or the femur. In severe cases this can include bone, but loss of the articular cartilage is more common (Figure 6). Imaging of these cartilaginous injuries requires MRI, which will also show the associated ligamentous injuries (typically rupture of the medial patellofemoral ligament) and marrow oedema adjacent to the site of injury (Figure 7). Developmental anomalies such as a shallow trochlear sulcus or patella alta (high-riding patella) predispose to dislocation and maltracking. These can be seen on X-rays, CT and

\section{Box 2. Australian Medicare Benefits Schedule criteria for knee magnetic resonance imaging ${ }^{18}$}

The Medicare Benefits Schedule allows patients to be referred by general practitioners for magnetic resonance imaging:

- following acute knee trauma for patients aged 16-49 years with inability to extend the knee suggesting the possibility of acute meniscal tear or clinical findings suggesting acute anterior cruciate ligament tear

- for a scan of the knee for a patient aged $<16$ years for internal joint derangement.
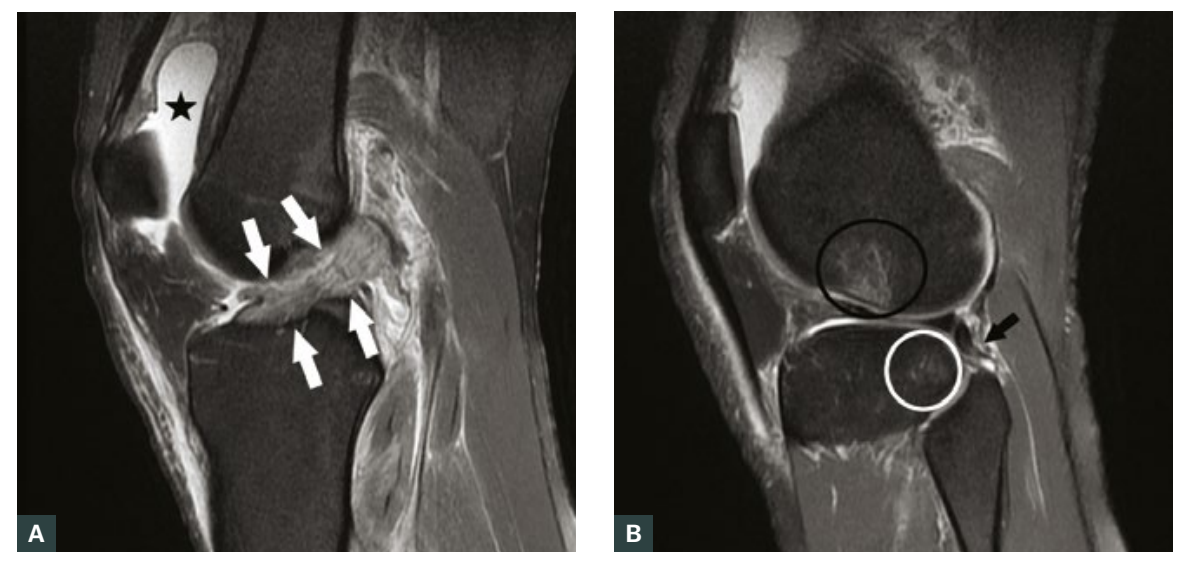

Figure 3. Sagittal proton density fat-saturated image

A. Complete anterior cruciate ligament tear (white arrows). Note also the knee joint effusion (black star); B. Microtrabecular fractures in posterolateral tibial plateau (white ring) and the lateral femoral condyle (black ring) typical of a 'pivot shift' injury. At the time of injury, these points impacted on each other, causing the bone injury. There is an associated posterolateral corner injury including a partial tear of the popliteofibular ligament (black arrow).

Note: These images are both of the same patient. 


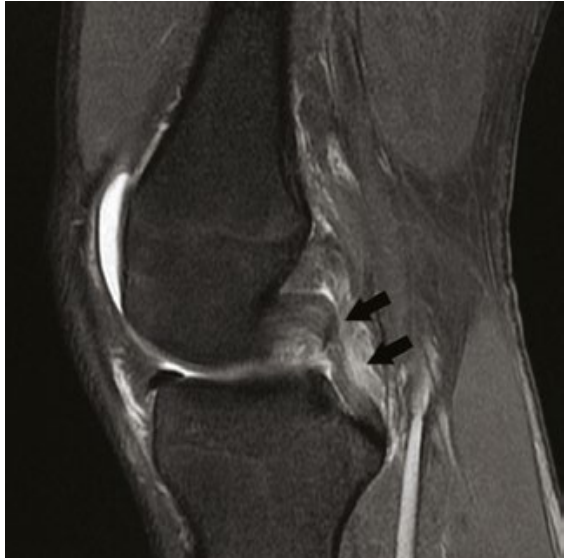

Figure 4. Sagittal proton density fat-saturated image showing ruptured posterior cruciate ligament (black arrows)

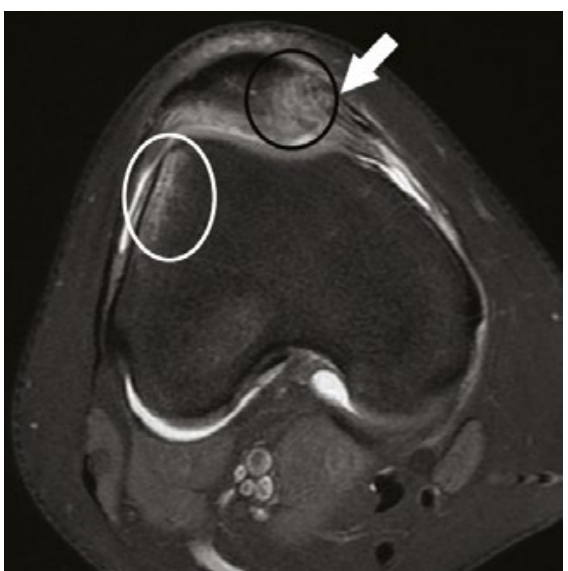

Figure 7. Axial proton density fat-saturated image from a girl aged 13 years with knee pain showing rupture of the medial patellar retinaculum (white arrow). Bone contusions in lateral femoral condyle (white circle) and medial patella (black circle) indicate that these two points impacted at the time of injury, showing that this represents an unrecognised transient patellar dislocation. The femoral trochlea is also shallow. There was also a patella alta (another predisposing factor for dislocation; not shown).

MRI. Recurrent dislocations increase the likelihood of cartilage loss. ${ }^{8}$

\section{Osteochondral defects}

Osteochondral defects are focal areas of damage of the cartilage and underlying bone. They are a common source of knee problems in children and young

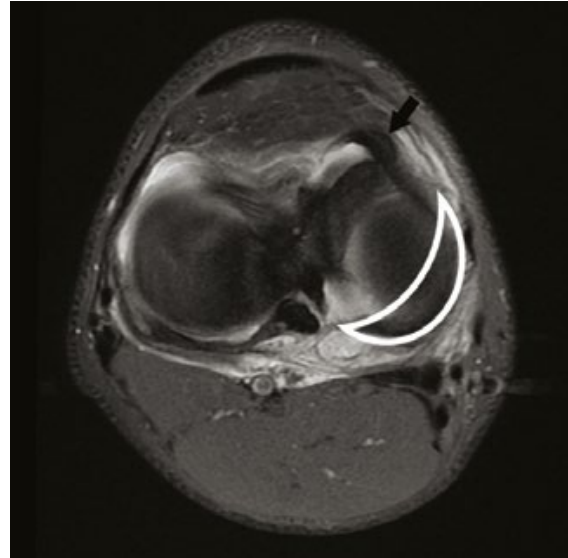

Figure 5. Axial proton density fat-saturated image showing abnormal location of the meniscus (black arrow) due to bucket handle tear. The normal position of the body and the posterior horn of the medial meniscus is shown by the white outline.

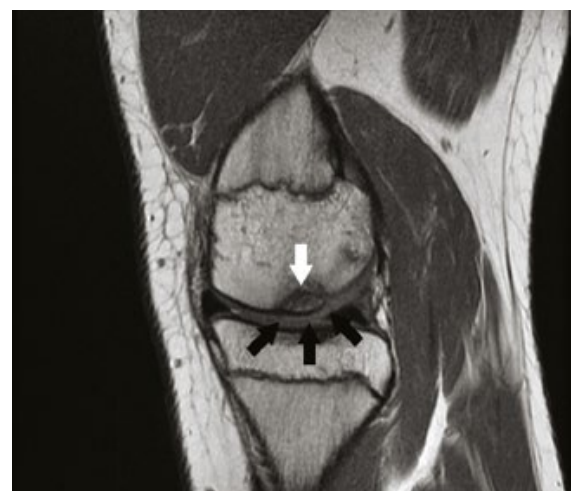

Figure 8. Sagittal proton density image showing an osteochondral defect in the medial femoral condyle with bone involvement (white arrow) and 'softening' of the cartilage as indicated by the change in signal (black arrows)

adults. Current theory for aetiology favours repetitive microtrauma with microtrabecular fractures of subchondral bone, subsequent ischaemia and altered local growth. ${ }^{9}$ In the early phase, when they are best treated, osteochondral defects can only be seen on MRI (Figure 8). In the late phase, which is more common with presentations in young adults, they can dislodge from the bone, leaving a defect in the articular surface and a loose body. These predispose to early osteoarthritis.

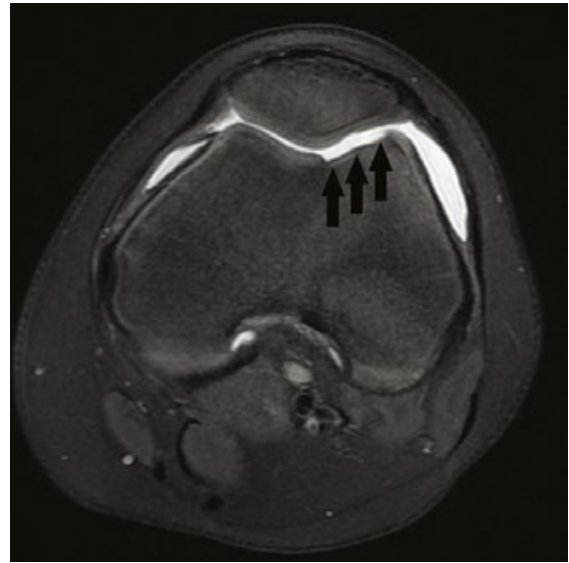

Figure 6. Axial proton density fat-saturated image showing a large defect in the articular cartilage of the femoral condyle from previous patellar dislocation (black arrows)

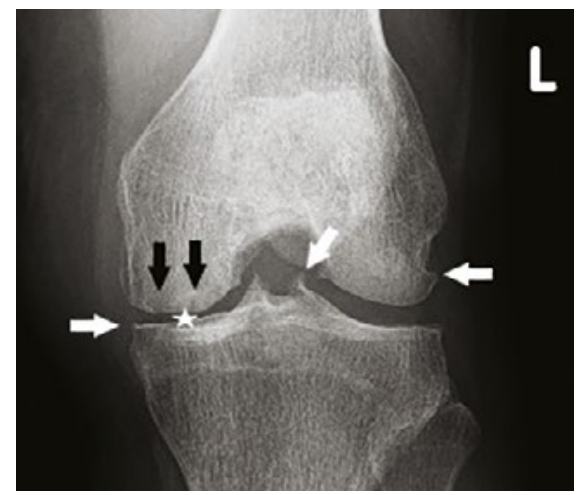

Figure 9. Osteoarthritis. Note the joint space narrowing in the medial compartment (white star), osteophytes (white arrows) and subchondral cysts (black arrows).

\section{Monoarticular arthritis}

Many forms of arthritis can present as an acute monoarticular arthritis. While studies of the aetiology of monoarticular arthritis are not specific to the knee, common differentials are gout (15-27\%), septic arthritis (8-27\%), osteoarthritis (5-17\%), rheumatoid arthritis (11-16\%) and pseudogout (calcium pyrophosphate deposition disease [CPPD]). ${ }^{10}$

Radiology has a role in characterising the type of arthritis and the extent of disease. 

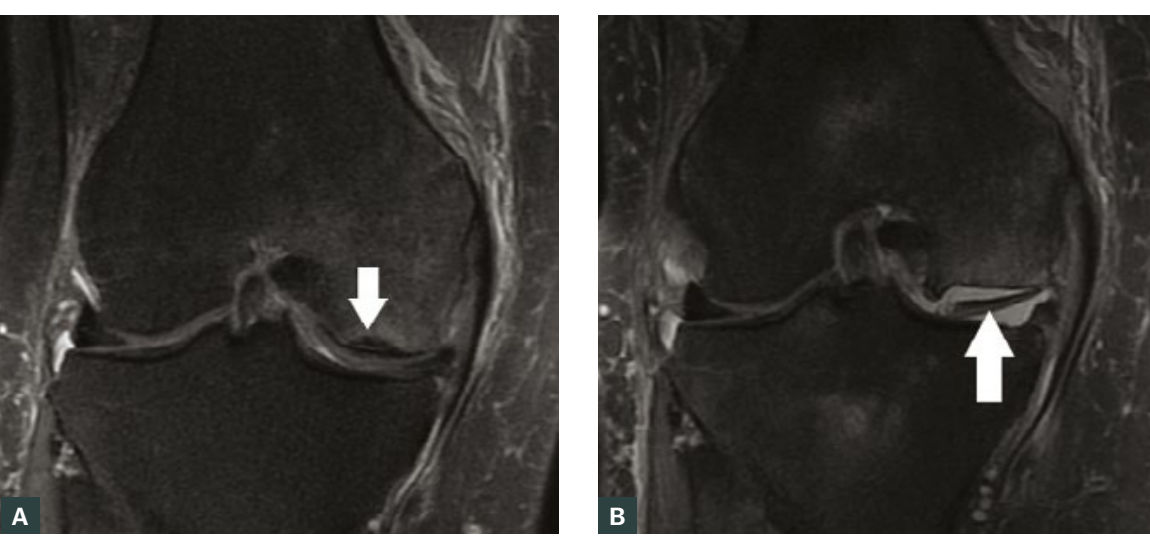

Figure 10. Coronal proton density fat-saturated image

A. Patient with osteoarthritis and new knee pain. There is a subchondral insufficiency fracture (white arrow); B. Two months later. Marked progression of the insufficiency fracture with loss of the femoral articular surface and a large fragment in the joint (white arrow).

Note: These images are both of the same patient.

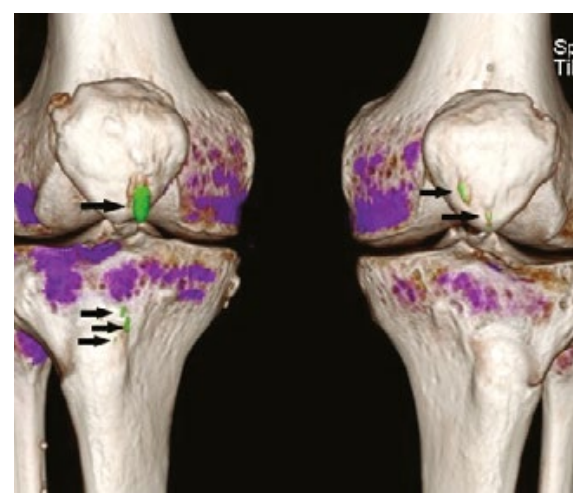

Figure 11. Volume-rendered dual-energy computed tomography scan. Urate deposits (gouty tophi) are shown in green (arrows). Other views show these to lie in the patella tendons bilaterally.

\section{Osteoarthritis}

Up to half of people over the age of 50 years report knee pain in any year, and a quarter of people will have severe and disabling pain. ${ }^{11}$ The rate of osteoarthritis increases with age. Previous knee injury is a major risk factor. Other risk factors for knee osteoarthritis include obesity, osteoarthritis in the hand and being female. ${ }^{11}$

Osteoarthritis is characterised by damage to, and loss of, cartilage. X-rays are the first-line investigation (Figure 9). Joint space narrowing is the pathognomonic

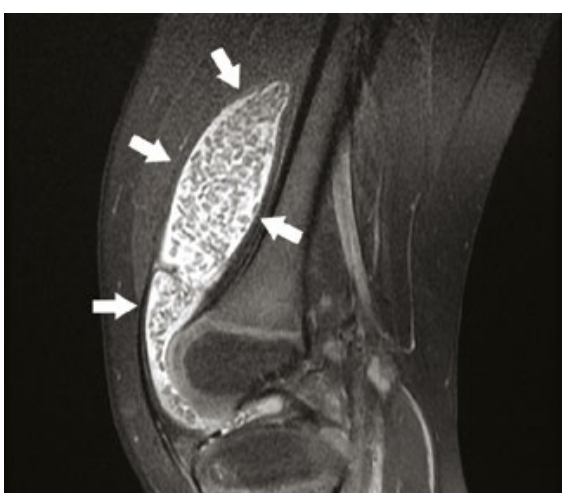

Figure 12. Sagittal proton density fat-saturated image of a patient, aged five years, with knee pain. Joint effusion (white arrows) with multiple filling defects representing synovial proliferation/inflammation. In this case, the diagnosis is juvenile idiopathic arthritis.

feature, although this is better shown on weight-bearing views. In some cases, the patellofemoral joint is more involved than the tibiofemoral compartments (eg secondary to abnormalities of patellar tracking). Other features of osteoarthritis include the presence of osteophytes and subchondral sclerosis. In more advanced cases, subchondral cysts and irregularity of the articular surface may be found. Joint effusions are also common.

The early cartilage changes of osteoarthritis will be seen even in younger patients (aged $<50$ years) who are having an MRI for other indications. These changes include partial cartilage loss, change in cartilage signal (sometimes described as 'softening') and fissures.

An X-ray is sufficient to diagnose osteoarthritis. Further imaging is dependent on whether there are clinical features suggesting acute complications that may warrant more specific management. These include subchondral insufficiency fractures and avascular necrosis, which are more common in elderly females (aged $>65$ years; Figures $10 \mathrm{~A}$ and 10B). ${ }^{12}$ Pain is associated with moderate-to-large effusions and synovial hypertrophy. Baker's cysts (a posterior extension of synovial fluid into the popliteal fossa) are present in $20 \%$ of patients with osteoarthritis. ${ }^{13}$

\section{Septic arthritis}

Septic arthritis is an important but uncommon condition that should always be considered in a monoarthritis as infection can cause devastating destruction of the joint. Risk factors include older age (>65 years), rheumatoid arthritis, diabetes, immunocompromise, previous knee surgery (especially prosthetic joints) and bacteraemia (eg from intravenous drug use or infection at a different site). Clinical features lack specificity, so awareness is critical. Presentations include an acutely painful joint. Fever was only found in $57 \%$ of cases in one series. ${ }^{14}$ Imaging, such as $\mathrm{X}$-ray or MRI, will usually show an effusion. In severe cases there is erosion of the bone. When there is suspicion of a septic joint, aspiration of synovial fluid is required for diagnosis. ${ }^{14}$

\section{Gout}

Diagnosis of gout is based on a score calculated from eight domains. These include characteristic radiological appearances (eg periarticular erosions and identification on dual-energy CT), clinical features and demonstration of raised urate levels in blood and synovial fluid. The presence of a monoarthritis in the foot or involvement of the first metatarsophalangeal joint in either monoarticular or polyarticular arthritis is included in the diagnostic criteria. ${ }^{15}$ Dual-energy CT is a relatively new 
technique able to directly visualise tophi, giving new insights into the distribution of tophi. It has shown, for example, that the knee is the second most common joint (after the foot) to have urate crystal deposition (Figure 11). ${ }^{16}$

\section{Rheumatoid arthritis}

Rheumatoid arthritis is characterised by synovial proliferation and synovitis. Typically, there will also be bone marrow oedema and bone erosions. X-ray changes (bone erosions and periarticular osteopenia) usually occur in more advanced disease. Ultrasonography and MRI can show synovial proliferation before the development of erosions (Figure 12). Bone marrow oedema is another important characteristic of rheumatoid arthritis on MRI, and it predicts erosive progression. ${ }^{17}$

\section{Conclusion}

The choice of imaging modality depends on the provisional diagnosis derived from clinical examination. X-rays are recommended as the initial test in trauma and arthritis. Superficial soft tissue injury can be evaluated with ultrasonography. Internal derangement, such as chondral, ligamentous or meniscal injury, can only be seen on MRI. In addition, MRI can also provide clues about the mechanism of injury from the bone marrow oedema pattern - for example in transient patellar dislocation, hyperextension injury or pivot shift injury.

\section{Authors}

Ursula Ridley MBBS (Hons), FRANZCR, Clinical Director, I-MED Radiology Network, NSW. ursula.ridley@gmail.com

Lloyd Ridley MBBS, FRANZCR, Senior Staff Specialist, Department of Radiology, Concord Hospital, NSW; Clinical Associate Professor, Discipline of Medical Imaging, University of Sydney, NSW Competing interests: None.

Funding: None.

Provenance and peer review: Commissioned, externally peer reviewed.

\section{References}

1. Bunt $C W$, Jonas CE, Chang JG. Knee pain in adults and adolescents: The initial evaluation. Am Fam Physician 2018;98(9):576-85.

2. Diagnostic Imaging Pathways. Diagnostic imaging pathways - knee pain (non-traumatic). Perth: Diagnostic Imaging Pathways, 2013.

3. Olsson O, Isacsson A, Englund M, Frobell RB. Epidemiology of intra-and peri-articular structural injuries in traumatic knee joint hemarthrosis Data from 1145 consecutive knees with subacute MRI. Osteoarthritis Cartilage 2016;24(11):1890-97. doi: 10.1016/j.joca.2016.06.006.

4. Yao K, Haque T. The Ottawa knee rules: A useful clinical decision tool. Aust Fam Physician 2012;41(4):223-24.

5. Scolaro J, Bernstein J, Ahn J. In brief: Patellar fractures. Clin Orthop Relat Res 2011; 469(4):1213-15. doi: 10.1007/s11999-010-1537-8.

6. Swamy GN, Nanjayan SK, Yallappa S, Bishnoi A, Pickering SA. Is ultrasound diagnosis reliable in acute extensor tendon injuries of the knee? Acta Orthop Belg 2012;78(6):764-70.

7. Montalvo AM, Schneider DK, Webster KE, et al. Anterior cruciate ligament injury risk in sport: A systematic review and meta-analysis of injury incidence by sex and sport classification. J Athl Train 2019;54(5):472-82. doi: 10.4085/10626050-407-16.

8. Endo Y, Stein BE, Potter HG. Radiologic assessment of patellofemoral pain in the athlete. Sports Health 2011;3(2):195-210. doi: 10.1177/1941738110397875.

9. Durur-Subasi I, Durur-Karakaya A, Yildirim OS. Osteochondral lesions of major joints. Eurasian J Med 2015;47(2):138-44. doi: 10.5152/ eurasianjmed.2015.50

10. Ma L, Cranney A, Holroyd-Leduc JM. Acute monoarthritis: What is the cause of my patient's painful swollen joint? CMAJ 2009;180(1):59-65. doi: 10.1503/cmaj.080183.

11. Blagojevic $M$, Jinks $C$, Jeffery $A$, Jordan KP. Risk factors for onset of osteoarthritis of the knee in older adults: A systematic review and metaanalysis. Osteoarthritis Cartilage 2010;18(1):24-33. doi: 10.1016/j.joca.2009.08.010.

12. Fox MG, Chang EY, Amini B, et al. ACR Appropriateness Criteria ${ }^{\circledR}$ Chronic Knee Pain. J Am Coll Radiol 2018;15(11S):S302-S12. doi: 10.1016/j.jacr.2018.09.016.

13. Hill CL, Gale DG, Chaisson CE, et al. Knee effusions, popliteal cysts, and synovial thickening: Association with knee pain in osteoarthritis. J Rheumatol 2001;28(6):1330-337.

14. Clerc O, Prod'hom G, Greub G, Zanetti G, Senn L. Adult native septic arthritis: A review of 10 years of experience and lessons for empirical antibiotic therapy. J Antimicrob Chemother 2011;66(5):1168-173. doi: 10.1093/jac/dkr047.

15. Neogi T, Jansen TL, Dalbeth $N$, et al. 2015 gout classification criteria: An American College of Rheumatology/European League Against Rheumatism collaborative initiative. Arthritis Rheumatol 2015;67(10):2557-68. doi: 10.1002/ art.39254.

16. Mallinson PI, Reagan AC, Coupal T, Munk PL, Ouellette $\mathrm{H}$, Nicolaou S. The distribution of urate deposition within the extremities in gout: A review of 148 dual-energy CT cases. Skeletal Radiol 2014;43(3):277-81. doi: 10.1007/s00256-013-1771-8.

17. Colebatch AN, Edwards CJ, Østergaard M, et al. EULAR recommendations for the use of imaging of the joints in the clinical management of rheumatoid arthritis. Ann Rheum Dis 2013;72(6):804-14. doi: 10.1136/ annrheumdis-2012-203158.

18. MBS online. Medicare benefits schedule. Canberra: Department of Health, 2019. Available at www9.health.gov.au/mbs/search. $\mathrm{cfm} ? \mathrm{q}=63560 \&$ Submit $=\&$ sopt $=S$ [Accessed 18 December 2019]. 